\title{
Flexible Piezoelectric 0-3 PZT-PDMS Thin Film for Tactile Sensing
}

\author{
Kiran kumar Sappati, Student Member, IEEE, Sharmistha Bhadra, Member, IEEE.
}

\begin{abstract}
In this paper, Lead Zirconate-Titanate (PZT)/Polydimethylsiloxane (PDMS) based composite is fabricated and characterized for its potential application as a flexible piezoelectric material. Thin films of the composite are prepared by dispersing different volume percentage of PZT particles in cross-linked PDMS matrix. Corona poling of the films is performed to increase the piezoelectric charge constant. $X$-ray diffraction results indicate that poling aligns the randomly oriented ferroelectric domains in the PZT particles along the poling axis. Piezoelectric voltage response and piezoelectric charge constant values before and after the poling show the improvement in piezoelectricity due to poling. The piezoelectric charge constant, $d_{33}$ and magnitude of relative permittivity of the films increase and flexibility of the films decrease with the increase of PZT particle volume percentage in the composite. Results show that for $28 \mathrm{v} \%$ PZT composite poled film a $\mathrm{d}_{33}$ of $78.33 \mathrm{pC} / \mathrm{N}$ was achieved with a Youngs modulus of around $10 \mathrm{MPa}$ and magnitude of relative permittivity of 10 . The film demonstrates effectiveness for tactile sensing by generating charges across the film proportional to the finger pressure applied on them. The easy fabrication process makes the composite a promising candidate to build flexible low cost tactile sensing devices.
\end{abstract}

Index Terms-Composites, Flexibility, Tactile, PDMS, Piezoelectricity, PZT, Thin film, Sensors

\section{INTRODUCTION}

Piezoelectric materials can convert mechanical energy into electrical energy and electrical energy to mechanical energy. They have wide range of applications in sensors, microphones, speakers, accelerometers, strain gauges, medical imaging, energy harvesting [1]. Piezoelectric sensors have attracted a lot of attention because they have the lowest power need among other kinds of sensors [2]. Most piezoelectric materials used in systems are based on ceramics such as Barium titanate $\left(\mathrm{BaTiO}_{3}\right)$, Lead titanate $\left(\mathrm{PbTiO}_{3}\right)$, Lithium niobate $\left(\mathrm{LiNbO}_{3}\right)$, and Lead zirconate titanate $\left(\mathrm{PbZr}_{x} \mathrm{Ti}_{1-x} \mathrm{O}_{3}, 0\right.$ $<\mathrm{x}<1)$ due to high piezoelectric charge constant, $\mathrm{d}_{33}$. For example, depending on the synthesis and processing conditions, $\mathrm{d}_{33}>300 \mathrm{pC} / \mathrm{N}$ for PZT, $\mathrm{d}_{33}>200 \mathrm{pC} / \mathrm{N}$ for $\mathrm{BaTiO}_{3}$ can be achieved. [3]-[5]. However due to their brittleness, they are not suitable for flexible applications and are weak in tension [6]. Piezoelectric polymer materials offer mechanical flexibility, biocompatibility and easy solution based processability. Moreover, low acoustic impedance

The authors are with Department of Electrical and Computer Engineering, McGill University, Montreal, QC H3A-0E9, Canada.

(email: kiran.sappati@mail.mcgill.ca, sharmistha.bhadra@mcgill.ca).Copyright (c) 2013 IEEE.

Personal use of this material is permitted. However, permission to use this material for any other purposes must be obtained from the IEEE by sending a request to pubs-permissions@ieee.org. makes them favorable for sensing in the environments like water, human tissue and other organic materials. They are being widely researched for acoustic transducer, implantable medical devices, non-volatile low voltage memories and sensors. Although piezoelectric polymers offer wide range of advantages, their $\mathrm{d}_{33}$ is low compared to ceramics. For example, polyvinylidene fluoride (PVDF) which is one of the most widely studied and used piezoelectric polymer has a $\mathrm{d}_{33}$ of 20 to $34 \mathrm{pC} / \mathrm{N}$ [7]-[11].

Piezoelectric composite materials have formed to combine the high piezoelectric charge coefficient and dielectric constant of piezo ceramics with the flexibility and low density of polymers. Numerous processing techniques have been investigated to form composites of different connectivities. Most of the works have investigated connectivities which are easier to form such as $0-3,1-3,2-2,3-1,3-3$. Here the first digit in the notation represents the connectivity of the electromechanically active ceramic (piezoelectric) phase, and the second digit refers to the connectivity of the electromechanically passive phase (polymer). For example, composites with 0-3 connectivity have randomly dispersed piezoelectric particles in the 3D polymer matrix [12]. A wide variety of piezoelectric ceramic nanoparticles have been used with compatible polymer matrices to produce some compelling nano piezocomposites (nPC) for sensors, energy harvesters, vibration dampers and actuators. [13]-[17] have reported nPCs where they used PDMS as polymer matrix and $\mathrm{BaTiO}_{3}$ nanoparticles, $\mathrm{BeFiO}_{3}$ nanoparticles, PZT nanowires, $\mathrm{NaNbO}_{3}$ nanowires, $\mathrm{LiNbO}_{3}$ nanowires, respectively. In [18], researchers used $\mathrm{PVC}$ polymer matrix with $\mathrm{BaTiO}_{3}$ nanowires to develop a nPC. In this work, we have reported fabrication of thin films of 0-3 PZT-PDMS piezoelectric composite, investigated their piezoelectric, crystalline and mechanical behaviour, and demonstrated their application for tactile sensing.

PZT is a widely used piezoelectric ceramic with high $d_{33}$ and dielectric constant value and has a spontaneous electric polarization in multi dimensions. PZT is physically strong, chemically inert, relatively inexpensive to manufacture, and one of the widely used piezoelectric ceramics in the world. Further, PZT can be doped with donor and acceptor impurities which further modifies its piezoelectric properties for tailor made applications [19]. Polydimethylsiloxane (PDMS) and its co-polymers are one of the widely used polymer matrices for nPCs due to their viscoelasticity and softness. Variation in chemical cross linking is used to tune the mechanical properties of PDMS [20], [21]. Ease of 
preparation, bio compatibility, chemical resistance, low cost, optical transparency and thermal stability of PDMS renders to its wide use. Although some researchers have explored composite made of PDMS and PZT nanostructures, most of them involve complicated high-cost and low-throughput fabrication processes [15], [22], [23] . The composite film reported in [22] was made from highly-ordered PZT hemispheres embedded in PDMS matrix. It involves complicated process of LangmuirBlodgett deposition of the PS beads and RF sputtering of PZT on the spheres. In [15], researchers fabricated a textile composed of aligned parallel PZT nanowires by electrospinning. Then they embedded the PZT textile in PDMS. In [23], researchers electrospun PZT nanofibers on electrodes and then embedded the electrodes and nanofibers in PDMS.

In these contexts, we report an easy fabrication of PZT-PDMS composite by dispersing PZT particles in PDMS. Although very few groups have researched easy fabrication PZT-PDMS composite, our work has achieved higher piezoelectric charge constant than the piezoelectric charge constants reported by these groups [24], [25]. Prepared composite films have a piezoelectric response scalable to the PZT content in the polymer matrix. The effect of poling on the piezoelectric property was investigated by measuring the open circuit voltage across the film during bending and by measuring induced charge on applying a compressive force. The effect of poling on crystalline character was examined using X-ray diffraction (XRD) analysis. The mechanical properties and relative permittivity of the films were obtained from tensile tests and impedance measurements, respectively. Finally, the potential of the films for tactile sensing was demonstrated.

\section{EXPERIMENTAL}

\section{A. Materials}

PZT particles of purity $99 \%$ with an average particle size of $560 \mathrm{~nm}$ were purchased from American elements, USA and PDMS (two part Dow corning Sylgard-184 silicone elastomer) was purchased from Paisley, Canada. All the chemicals were used as received. The morphology of PZT particles was observed with FESEM (Field Emission Scanning Electron Microscope). Fig. 1 shows the FESEM image of PZT particles exhibiting a large variation in their shape and size.

\section{B. Fabrication of piezoelectric composite}

Fig. 2.a shows the schematic of the composite film preparation. At first, the PDMS solution was prepared by adding curing agent to the base. The volume ratio of the base to curing agent was 10:1. Then the PZT particles were dispersed into the PDMS solution at various concentrations: 3.5, 8.2, $11.3,13.3$ and $28 \mathrm{v} \%$. For each v\% of PZT particles, the total PZT particles were divided in three equal portions. After one portion was added, the mix was stirred for few minutes before adding the next portion. This helped with uniform dispersion of PZT particles in PDMS. After the third portion was added, the solution was stirred for 15 minutes. As shown in Fig. 2.a,

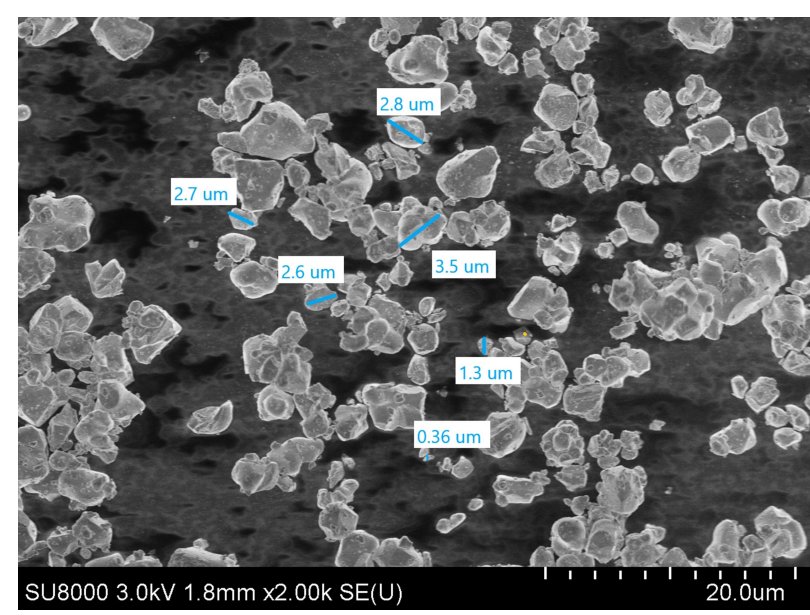

Fig. 1. FESEM image of PZT particles.
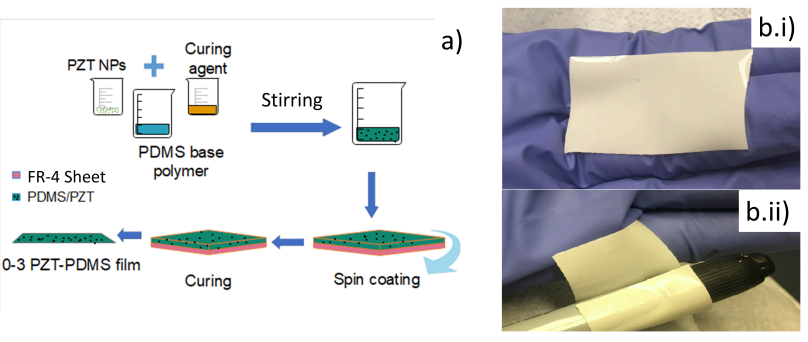

Fig. 2. a) Schematic of the composite film fabrication; b) Isolated composite film with $13.3 \mathrm{v} \%$ PZT after peeling off.

after mixing, the viscous solution was spin coated on to a FR4 board at $1000 \mathrm{rpm}$ for 15 seconds. As the viscosity changed with the PZT content in PDMS, there was a slight variation in the thickness of films for different PZT v\% films. Measured thicknesses of all the films were in between 130-165 microns. Following the spin coating, the films were left to cure in the oven at $120^{\circ} \mathrm{C}$ for 20 hours. After curing, the films were peeled off from the FR-4 substrate and cut into the sizes of $5 \times 2.5$ $\mathrm{cm}$ for corona poling. Since FR-4 is made of non-conductive glass fiber, the cured PDMS can be peeled off easily with little dexterity. Fig. 2.b, shows the softness and flexibility of 11.3 $\mathrm{v} \%$ isolated PZT film.

FESEM of different $\mathrm{v} \%$ of PZT films was carried out by high resolution SEM, Hitachi model SU8000. Fig. 3 shows the cross section of the $28 \mathrm{v} \%$ PZT film. It can be seen that the PZT particles are uniformly distributed in PDMS. It is important to note that PZT particles are heavier. Therefore, they tend to agglomerate at high concentrations in the polymer. Even in our case we observed small agglomerated particles at $28 \mathrm{v} \%$ PZT. Agglomeration can be reduced by dispersing the ceramic particles in a solvent such as toluene before adding it to the polymer matrix [26]. 


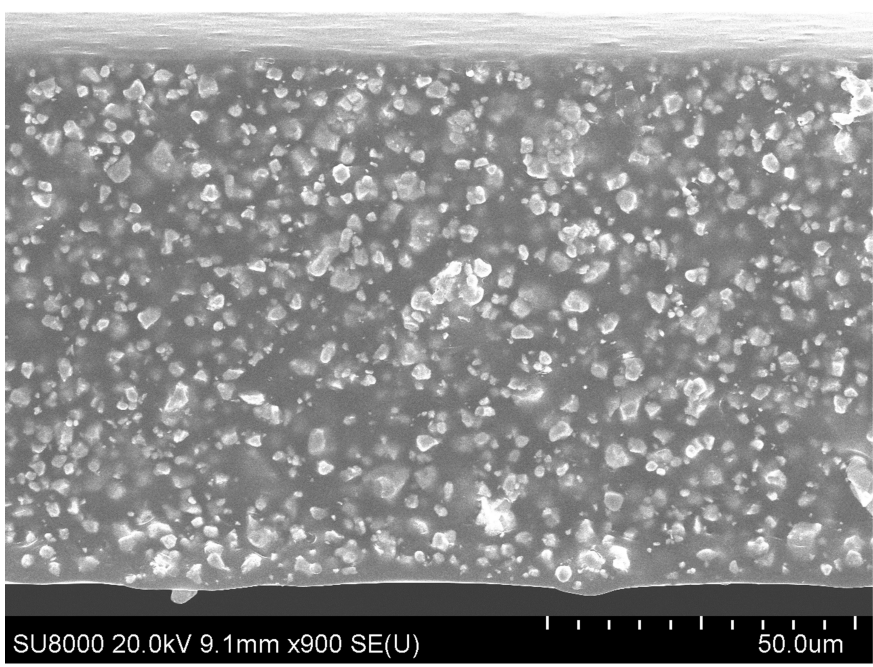

Fig. 3. FESEM image of cross-section of $28 \mathrm{v} \%$ PZT film.
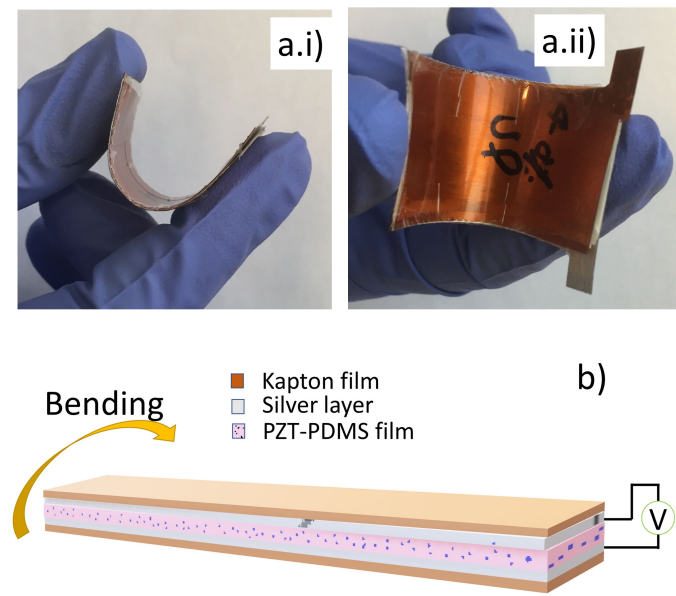

Fig. 4. Packaging of piezocomposite films. a) Image of a packaged film showing the flexibility of the packaging b) Schematic of the packaged composite film.

Corona poling causes the aligning of ferroelectric domains in the PZT particles in the direction of electric field. This eventually increases the piezoelectricity [27], [28]. Corona poling is advantageous over contact poling because large area samples can be poled without contact electrodes which minimizes the chance of dielectric breakdown of the sample. The 0-3 PZT-PDMS films were corona poled using an applied electric filed of $9 \mathrm{kV} / \mathrm{mm}$ at a temperature of $120^{\circ} \mathrm{C}$ for 2 hours in air. Resistivity of the polymers is much higher than the ceramic particles. During corona poling, piezoelectric composites are heated to decrease the resistivity of the polymer so that the voltage drop across the ceramic particle increases. This increases the poling efficiency [29].
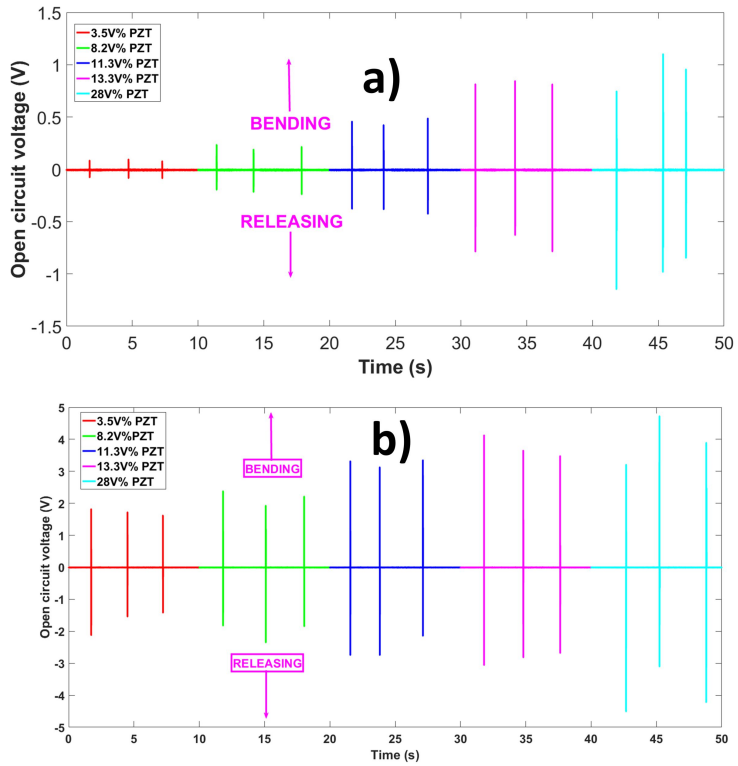

Fig. 5. Open circuit voltage response across the thickness of the films for bending and release of a) unpoled and b) poled films.

\section{Characterization And Results}

\section{A. Piezoelectric voltage response}

The piezoelectric response of the films was observed by measuring the open circuit voltage generated across the thickness of the films when subjected to bending and releasing motions. Because of the hydrophobic nature of composite surface due to PDMS, it is difficult to adhere the metal inks to composite [30], [31]. To have a better metal contact to the composite surface, conductive silver ink (Voltera-Flex conductor) was coated on one side of the thin Kapton (polyimide) films (125 micron thick) and the PZT-PDMS films were packaged as a sandwich in between these silver coated kapton films as shown in Fig. 4.a and b. Before sandwiching the composite films in between the Kapton films, the ink was cured in oven at $180^{\circ} \mathrm{C}$ for 30 minutes. The Kapton films with silver electrodes were flexible and offered easy bending and releasing for the packing while providing good metallic contacts to the composite surfaces. Bending and releasing with fingers were performed to create agile mechanical moments on the films. By virtue of piezoelectric nature, the films produced voltage across the thickness which clearly corresponded to the bending and release motions. These voltage responses were captured with the NI, USB-6009 serial data acquisition interface. The open circuit voltage produced across the thickness of the film is a function of piezoelectric charge constant $\left(d_{15}\right)$ and Young's modulus(Y) [32] given by

$$
V_{o c}=\int g_{15} x(l) Y d l
$$

Here, $g_{15}$ is the piezoelectric strain constant which equals to $\mathrm{d}_{15} / \epsilon$ and $\mathrm{x}(\mathrm{l})$ is elastic strain produced along the thickness ' 1 ' and $\epsilon$ is the absolute permittivity of the composite.

Fig. 5 shows the piezoelectric voltage responses of the poled and unpoled films with different $v \%$ PZT during bending and release motions. Here, positive and negative peaks correspond 
to bending and release motions, respectively. Maximum voltage responses observed for unpoled films were $0.095,0.23$, $0.49,0.84$, and $1.14 \mathrm{~V}$ for $3.5,8.2,11.3,13.3$ and $28 \mathrm{v} \%$ PZT, respectively. Maximum voltage responses for poled films were $2.13,2.37,3.34,4.12$ and $4.7 \mathrm{~V}$ for $3.5,8.2,11.3,13.3$ and $28 \mathrm{v} \%$ PZT, respectively. Achieved responses show that the output voltage of the poled composite film is at least 4 times higher than the unpoled films for all v\% of PZT. This demonstrates that the output is associated with the piezoelectricity of PZT-PDMS composite and corona poling essentially improves the piezoelectricity of the composite. Results also show that the piezoelectricity of the composite increases with the increase of $\mathrm{v} \%$ of PZT. However, the flexibility of the composite decreases with the increase of $\mathrm{v} \%$ of PZT.

\section{B. XRD results}

For the crystalline characterization of PZT-PDMS films, we employed a Bruker D8 Discovery X-Ray Diffractometer with a $\mathrm{Cu}$ Source of $\mathrm{K} \alpha$ radiation, $1.54060 \mathrm{~A}^{0}$. The XRD results showed that the dispersed PZT particles in PDMS have a tetragonal structure with lattice parameters $\mathrm{a}=4.03466 \mathrm{~A}^{0}$, $\mathrm{c}=4.13262 \mathrm{~A}^{0}$. We performed the XRD on same location of the film before and after the poling. Fig. 6.a and b show the XRD spectrum for [001] and $c$ and $d$ show the [002] and [200] orientations of one sample of $13.3 \mathrm{v} \%$ and two samples of $30 \mathrm{v} \%$ PZT composite films, respectively before and after poling. It can be seen that the XRD curves for two samples of $30 \mathrm{v} \%$ PZT are very similar showing the repeatability of the poling process. During corona poling, the ferroelectric domains in PZT particles try to align with the applied electric field which causes a orientation change in their texture. It can be seen from Fig. 6, after poling, the average peak intensity increased for [001] and [002] orientation and decreased for [200] orientation. After poling, the orientation distribution of ferroelectric domains in PZT particles changes to a new stable state. However, due to dielectric relaxation of the PZT particles in PDMS matrix, the intensity difference of diffraction peaks after poling starts decreasing with time. In these experiments, the XRD was performed several hours after the poling which resulted in small difference of the XRD diffraction peaks before and after poling. Although the difference of peak intensity before and after poling is small, the same trend was observed for all the films. On an average, the ratio of $002 / 200$ peaks for $13.3 \mathrm{v} \%$ poled films has improved to $64.72 \%$ from a ratio of $56.42 \%$ for unpoled films. For 30 $\mathrm{v} \%$ poled films, the average ratio of $002 / 200$ peaks improved to $62.5 \%$ from a ratio of $48.12 \%$ for the unpoled films. This indicates that the effect in the tetragonal PZT particles is associated with increase in [001] and [002] orientation [29], [33]. Preferably, the ferroelectric domains change their random orientation mostly to [001] direction. From these results, it can be understood that the poling aligns the randomly oriented ferroelectric domains in PZT particles in the direction parallel to the applied electric field which improves the piezoelectric charge constant predominantly, $\mathrm{d}_{33}$.
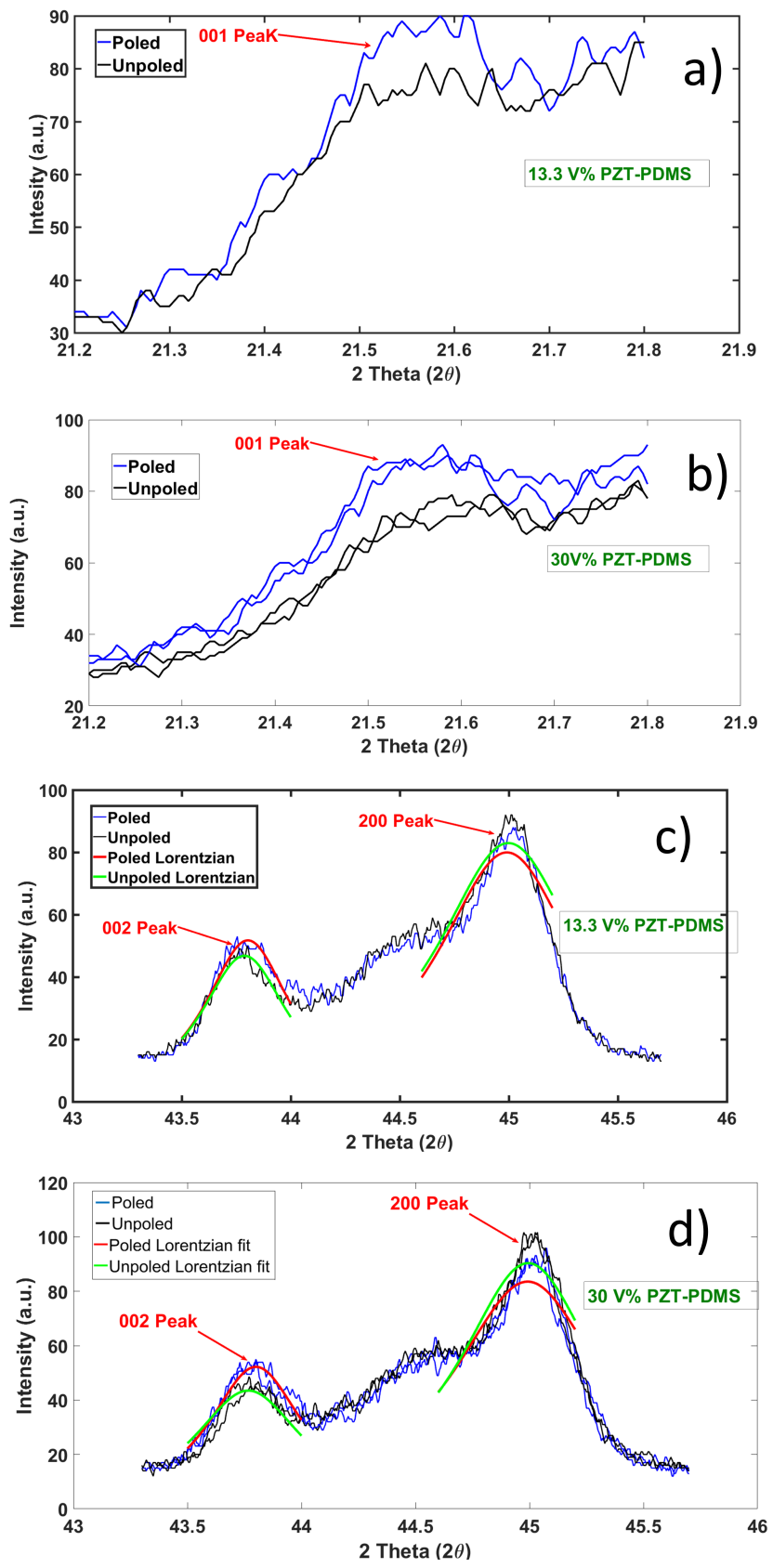

Fig. 6. XRD spectrum of 13.3 and $30 \mathrm{v} \%$ PZT composite film samples before and after poling; $a$ and b) XRD spectrum of [001] orientation of 13.3 and 30 $\mathrm{V} \%$ respectively; $\mathrm{c}$ and d) XRD spectrum of [002] and [200] orientations of 13.3 and $30 \mathrm{v} \%$ respectively.

\section{Dielectric properties}

The highly adaptable dielectric properties of piezoelectric composites is advantageous for making them suitable for applications like transducers, hydrophones, sonars, and storage capacitances [34], [35]. In a piezoelectric composite, dielectric permittivity is a function of volume fraction, ceramic particle size and temperature [36]. The relative permittivity of the prepared films was measured with an LCR meter (Keysight E4890A) using parallel plate method. Relative permittivity, $\epsilon_{r}$ 


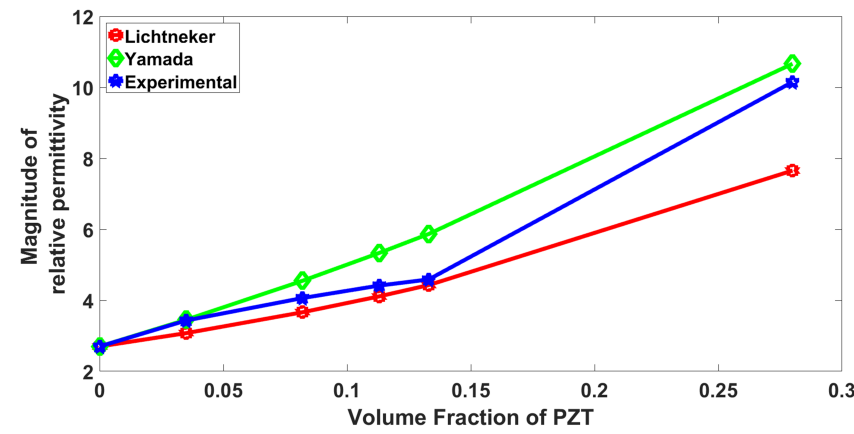

Fig. 7. Comparison of measured dielectric constant of all PZT v\% with theoretical models.

was determined by the equation.

$$
\epsilon_{r}=\frac{C_{p}}{C_{0}}-j \frac{G}{\omega C_{0}}
$$

where $\mathrm{C}_{p}$ and $\mathrm{G}$ are is the measured parallel plate capacitance and conductance of the PZT-PDMS film and $\mathrm{C}_{0}$ is the capacitance of air for the same area and thickness.

Theoretical models such as Maxwell-Wagner, Rayliegh's, Furukawa and Bhimasankaram (BSP) are used for prediction of relative permittivity of piezoelectric composites. Most of these models consider spherical particles for filler material. For our analysis, we have considered Modified Lichtnecker and Yamada models as each of them allows some leverage to the particle shape with fitting factors. Fitting factor also accounts for the interaction between the filler and matrix. Fig. 7 provides the comparison between the experimentally measured relative permittivity values with the theoretical models. A fitting factor of 0.3 for Lichtnecker and 8 for Yamada are best suited for our results [37]. It can be seen that the measured values were closer to the theoretical values for lower concentration of PZT compared to the higher concentration of PZT due to inferior dispersion at high concentrations.

It can be seen that magnitude of the relative permittivity increases with $\mathrm{v} \%$ of the PZT in the composite. This shows that the composites can be tailored for various dielectric constant requirements.

\section{Piezoelectric properties}

The piezoelectric properties of PZT-PDMS films were studied by measuring the charge induced across the films when subjected to a compressive force. After packing with kapton electrodes, the poled and unpoled films were placed on Electroforce-5500 machine and step forces were applied across the thickness of the films. Kistler 5015 Charge meter was employed for measuring the induced charge against corresponding force. Fig. 8 shows the charge response of poled and unpoled films for a step force of $1 \mathrm{~N}$. This curve clearly indicates the developed charge difference between poled and unpoled for all v\% PZT films. Another experiment was carried out on all poled films where increasing step forces, i.e, 0.5 , 1 and $1.5 \mathrm{~N}$ were applied on the films and charges were measured across the films. The results are shown in Fig. 9.
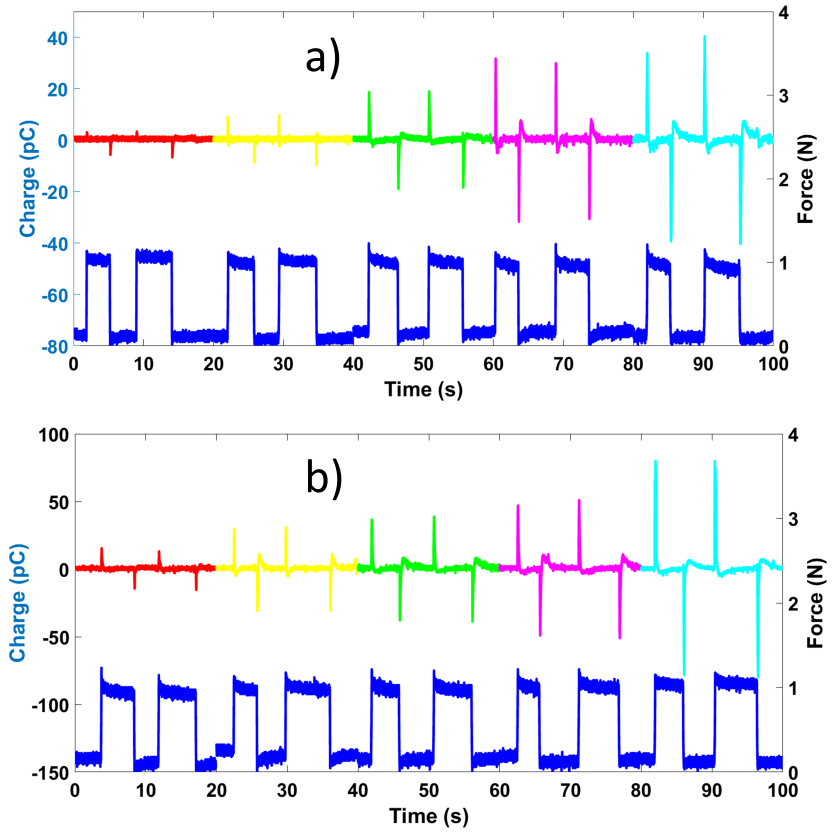

Fig. 8. Charge response of a) unpoled and b) poled films for a step force of $1 \mathrm{~N}$. Red, Yellow, Green, Magenta and Cyan indicates 3, 8, 11, 13 and 28 v\% PZT, respectively.

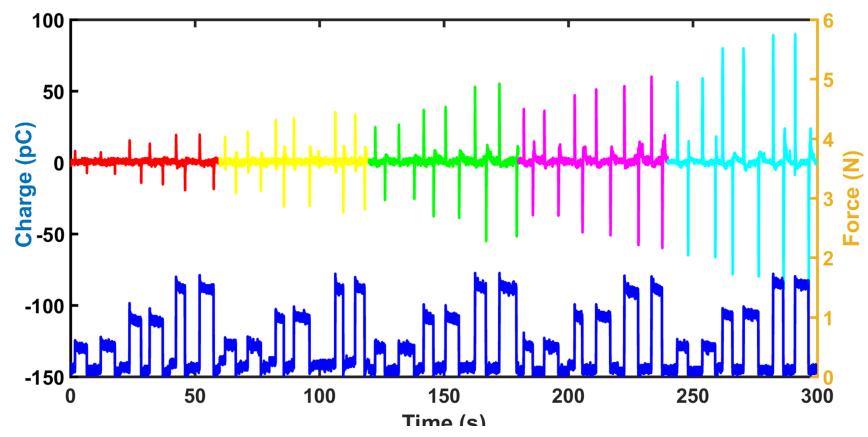

Fig. 9. Charge response of all $\mathrm{v} \%$ PZT poled films against variation of force.Red, Yellow, Green, Magenta and Cyan indicates 3, 8, 11, 13 and 28 $\mathrm{v} \%$ PZT, respectively.

The charge response is proportional to the amount of force applied and $\mathrm{v} \%$ PZT. The same curve is used to calculate $\mathrm{d}_{33}$ using the following equation [38].

$$
d_{33}=\frac{\text { Total Induced Charge }}{\text { Total force }} .
$$

The $d_{33}$ values obtained by the above equation are tabulated in Table I for all $\mathrm{v} \%$ PZT films.For the $28 \mathrm{v} \%$ PZT film , a d 33 of $78.33 \mathrm{pC} / \mathrm{N}$ was achieved. For similar v\% of PZT, the $\mathrm{d}_{33}$ obtained in our work is higher than the $\mathrm{d}_{33}$ reported for PZTPDMS composites prepared following an easy process. [24] reported a $\mathrm{d}_{33}$ of around $8 \mathrm{pC} / \mathrm{N}$ for $30 \mathrm{v} \%$ PZT and [25] reported a $\mathrm{d}_{33}$ of around $11 \mathrm{pC} / \mathrm{N}$ for $35 \mathrm{v} \%$ PZT composites. Higher $\mathrm{d}_{33}$ achieved in our work may be attributed to the larger size of PZT particles [39] and an efficient poling process.

Another important observation is that the charge response saturates at a certain low compressive force producing a non- 
TABLE I

PIEZOELECTRIC CONSTANTS EVALUATED FOR ALL PERCENTAGE VOLUME OF PZT

\begin{tabular}{ll}
\hline $\mathrm{v} \%$ PZT & $\mathrm{d}_{33}(\mathrm{pC} / \mathrm{N})$ \\
\hline 3 & 14 \\
8 & 29.66 \\
11.3 & 40.33 \\
13.3 & 49.3 \\
28 & 78.33 \\
\hline
\end{tabular}

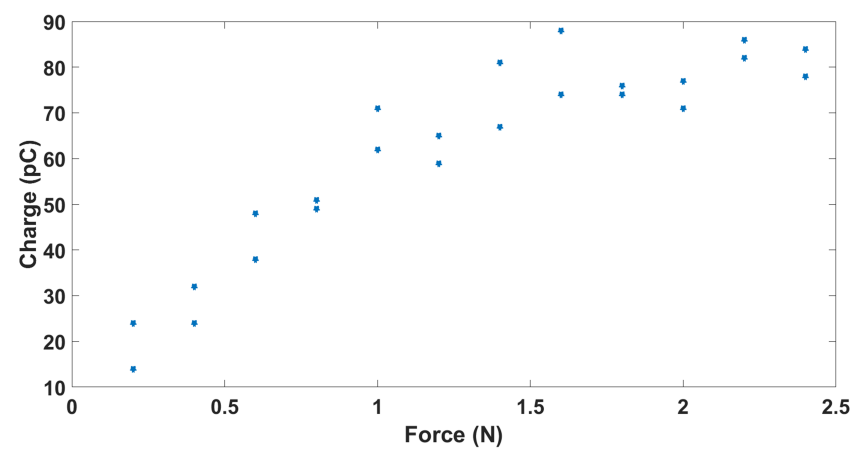

Fig. 10. linearity of charge response of a $28 \mathrm{v} \%$ PZT poled film for varying finger pressure.

linear behavior. This may be attributed to the maximum piezoelectric strain, $x(1)$ achieved at a low force because of the small thickness (around 150 microns) of films. In order to measure higher forces the thickness of the films need to be increased. We captured the non linearity in the charge response of $28 \mathrm{v} \%$ poled film by varying the step force between $0-2.5 \mathrm{~N}$ with the Electroforce machine. The charge response is plotted in Fig. 10. The key observation is that the charge response saturates above a certain force for these films, say above 1.5 $\mathrm{N}$ for the $28 \mathrm{v} \%$ films.

\section{E. Mechanical characterization}

The elastomeric nature of the PZT-PDMS composite can be critical to device performance in tactile sensor operations. In order to study the mechanical behaviour of the PZT-PDMS films, tensile tests were preformed on all v\% films using an Instron mini 44 series testing system. Samples were prepared in sizes of $35 \times 5 \mathrm{~mm}$ size and mounted between the jaws of the testing machine which were set to move apart at 6 $\mathrm{mm} /$ minute. The tensile test results are shown in Fig. 11. It can be observed that the tensile strength decreases as the concentration of PZT powder increases. The unevenness in the break points of increasing PZT v\% is due to the small variation in thickness and sample size. Further, the inset of Fig. 11 indicates that the initial slope of all curves is proportional to the $\mathrm{v} \%$ PZT.

Young's modulus values of the samples were calculated from the initial slope of tensile stress vs strain plots. Table II shows the Young's Modulus values for films with different percentage volume of PZT. As the percentage volume of PZT in the composite increases the Young's modulus increases. High Young's modulus results in effective transfer of stress and improvement in the open circuit voltage as explained in

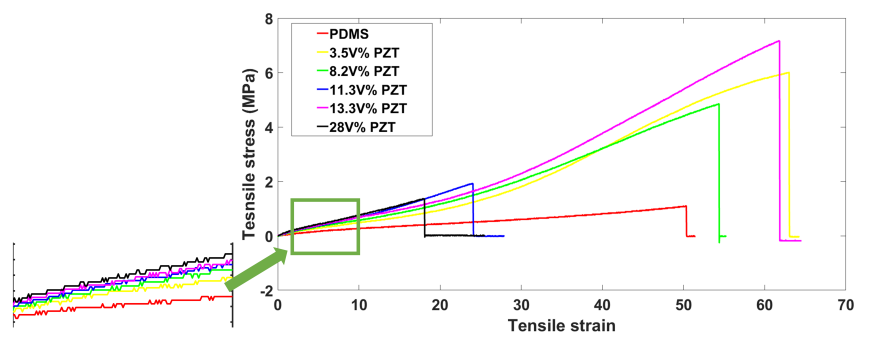

Fig. 11. Tensile test results for different volume percentage of PZT-PDMS films

TABLE II

YOUNG'S MODULUS VALUES FOR FILMS WITH DIFFERENT PERCENTAGE VOLUME OF PZT

\begin{tabular}{ll}
\hline v\% PZT & Y modulus (MPa) \\
\hline 0 & 4 \\
3 & 7.098 \\
8 & 7.97 \\
11.3 & 8.98 \\
13.3 & 8.69 \\
28 & 9.92 \\
\hline
\end{tabular}

Eq. 1. Results indicate that $28 \mathrm{v} \%$ PZT can be used as flexible film for tactile sensors.

\section{F. Tactile sensing}

To demonstrate the tactile sensing capability of our piezoelectric composite film, a 28 v\% PZT film was sandwiched between Kapton substrates with electrodes and placed on a reference force sensor (Force Sensor integrated in the Electroforce 5500 machine). The force exerted on the piezoelectric composite film due to the finger pressure was measured using the reference force sensor, while the response of the piezoelectric film was measured using the charge meter (Kistler 5015A). Care was taken to ensure that there is no electromagnetic interference due to finger touch by wearing insulating gloves and reducing the time constant of input charge amplifier unit in charge meter to the minimum. Moreover, the Kapton films are sufficiently insulating. Therefore, the charge from human hands cannot transfer to the films and the silver layer. Fig. 12 shows the piezoelectric response of composite film for four touch events along with the corresponding force exerted measured by force sensor. It can be seen that the magnitude of the charge induced across the film highly depends on the magnitude of the force exerted on each touch. For example, for the first event the charge induced by the paper is $141 \mathrm{pC}$ (force exerted is $0.75 \mathrm{~N}$ ) and it produced a lower charge for a smaller tactile pressure (second touch, $115 \mathrm{pC}$ at $0.7 \mathrm{~N}$ ). The film showed a similar signal amplitude for the third and the fourth event as well. This indicates that, while each tactile event leads to a sensor signal, the signal amplitude is directly proportional to the force applied on the film.

\section{COnClusion And Discussion}

A simple and low cost fabrication process is reported to produce 0-3 PZT-PDMS thin films. The fabrication approach 


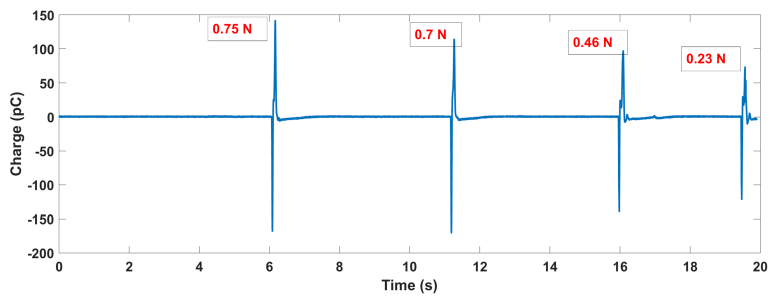

Fig. 12. Charge response of all $28 \mathrm{v} \%$ PZT poled films against finger pressure.

utilizes PZT particles and PDMS as base materials and the process involves dispersing of PZT particles in PDMS matrix by stirring, spin coating and curing. Results of FESEM show that the dispersion of the PZT particles is uniform and stable. Corona poling is performed for aligning the dipoles in ceramic particles and thus improving piezoelectric response. Piezoelectric response and piezoelectric charge constant, $\mathrm{d}_{33}$ measurements and XRD studies of the films before and after poling suggest that poling successfully aligns the randomly oriented ferroelectric domains in PZT particles and increases the piezoelectricity of the films. Piezoelectric charge constant, relative permittivity of the films increase and flexibility of the films decreases with increase of PZT volume percentage in the composite. The $28 \mathrm{v} \%$ PZT composite poled film achieves a piezoelectric charge constant of $78.33 \mathrm{pC} / \mathrm{N}$ with a Youngs modulus of $10 \mathrm{MPa}$ and relative permittivity magnitude of 10 . It produces an open circuit voltage of $4.7 \mathrm{~V}$ under bending and releasing by fingers.

Curie point of some PZT types such as PZT-4, PZT-5A and PZT-8 are above $300^{\circ} \mathrm{C}$ [40]. With high thermal stability of PDMS (around $300^{\circ} \mathrm{C}$ [41]) PZT-PDMS composites can be made for high temperature applications. Response of PZT particles varies with temperature. The same is evident For example, the resonant frequency of PZT discs changes with temperature. Our composite films will have response changes due to temperature variations as well. In order to address the variations of sensor performance due to temperature changes, various temperature compensation techniques using statistical methods such as data normalization technique using Kernel principal component analysis (KPCA), Hilbert transform method can be employed [42], [43].The piezoelectric and dielectric properties of the composite films are better than piezoelectric polymers, such as PVDF. When compared with piezoelectric polymers such as PVDF with Young's modulus of $2.5 \mathrm{GPa}$, these composite films are highly flexible with lower Young's modulus which makes them favourable for flexible tactile sensing applications such as robotic e-skin. The proposed films can be an ideal candidate for low cost flexible tactile sensing.

\section{ACKNOWLEDGMENT}

The authors thank Ms Stephanie Bessette from McGill University for assistance with FESEM and Prof. Theodorus G.M. van de Ven group from McGill University for the tensile test facility. This project was supported by Natural Sciences and Engineering Research Council of Canada.

\section{REFERENCES}

[1] V. F. Janas and A. Safari, "Overview of fine-scale piezoelectric ceramic/polymer composite processing," Journal of the American Ceramic Society, vol. 78, no. 11, pp. 2945-2955, 1995.

[2] G. Park, T. Rosing, M. D. Todd, C. R. Farrar, and W. Hodgkiss, "Energy harvesting for structural health monitoring sensor networks," Journal of Infrastructure Systems, vol. 14, no. 1, pp. 64-79, 2008.

[3] T. Karaki, K. Yan, T. Miyamoto, and M. Adachi, "Lead-free piezoelectric ceramics with large dielectric and piezoelectric constants manufactured from batio3 nano-powder," Japanese Journal of Applied Physics, vol. 46, no. 2L, p. L97, 2007.

[4] H. Takahashi, Y. Numamoto, J. Tani, K. Matsuta, J. Qiu, and S. Tsurekawa, "Lead-free barium titanate ceramics with large piezoelectric constant fabricated by microwave sintering," Japanese journal of applied physics, vol. 45, no. 1L, p. L30, 2005.

[5] S. Wada, K. Yako, H. Kakemoto, T. Tsurumi, and T. Kiguchi, "Enhanced piezoelectric properties of barium titanate single crystals with different engineered-domain sizes," Journal of Applied Physics, vol. 98, no. 1, p. 014109, 2005

[6] H. Gullapalli, V. S. Vemuru, A. Kumar, A. Botello-Mendez, R. Vajtai, M. Terrones, S. Nagarajaiah, and P. M. Ajayan, "Flexible piezoelectric zno-paper nanocomposite strain sensor," small, vol. 6, no. 15, pp. 16411646, 2010.

[7] Z. Hu, M. Tian, B. Nysten, and A. M. Jonas, "Regular arrays of highly ordered ferroelectric polymer nanostructures for non-volatile low-voltage memories," Nature materials, vol. 8, no. 1, p. 62, 2009.

[8] F. S. Foster, K. A. Harasiewicz, and M. D. Sherar, "A history of medical and biological imaging with polyvinylidene fluoride (pvdf) transducers," IEEE transactions on ultrasonics, ferroelectrics, and frequency control, vol. 47, no. 6, pp. 1363-1371, 2000.

[9] G. R. Harris, R. C. Preston, and A. S. DeReggi, "The impact of piezoelectric pvdf on medical ultrasound exposure measurements, standards, and regulations," IEEE transactions on ultrasonics, ferroelectrics, and frequency control, vol. 47, no. 6, pp. 1321-1335, 2000.

[10] F. Wang, M. Tanaka, and S. Chonan, "Development of a pvdf piezopolymer sensor for unconstrained in-sleep cardiorespiratory monitoring," Journal of intelligent material systems and structures, vol. 14, no. 3 , pp. 185-190, 2003.

[11] S. Lee, E. V. Bordatchev, and M. J. Zeman, "Femtosecond laser micromachining of polyvinylidene fluoride (pvdf) based piezo films," Journal of Micromechanics and Microengineering, vol. 18, no. 4, p. 045011, 2008.

[12] R. Newnham, D. Skinner, and L. Cross, "Connectivity and piezoelectricpyroelectric composites," Materials Research Bulletin, vol. 13, no. 5, pp. 525-536, 1978.

[13] K.-I. Park, M. Lee, Y. Liu, S. Moon, G.-T. Hwang, G. Zhu, J. E. Kim, S. O. Kim, D. K. Kim, and Z. L. Wang, "Flexible nanocomposite generator made of batio3 nanoparticles and graphitic carbons," Advanced Materials, vol. 24, no. 22, pp. 2999-3004, 2012.

[14] X. Ren, H. Fan, Y. Zhao, and Z. Liu, "Flexible lead-free bifeo3/pdmsbased nanogenerator as piezoelectric energy harvester," ACS applied materials \& interfaces, vol. 8, no. 39, pp. 26 190-26197, 2016.

[15] W. Wu, S. Bai, M. Yuan, Y. Qin, Z. L. Wang, and T. Jing, "Lead zirconate titanate nanowire textile nanogenerator for wearable energyharvesting and self-powered devices," ACS nano, vol. 6, no. 7, pp. 62316235, 2012.

[16] J. H. Jung, M. Lee, J.-I. Hong, Y. Ding, C.-Y. Chen, L.-J. Chou, and Z. L. Wang, "Lead-free nanbo3 nanowires for a high output piezoelectric nanogenerator," ACS nano, vol. 5, no. 12, pp. 10041-10 046, 2011.

[17] B. K. Yun, Y. K. Park, M. Lee, N. Lee, W. Jo, S. Lee, and J. H. Jung, "Lead-free linbo 3 nanowire-based nanocomposite for piezoelectric power generation," Nanoscale research letters, vol. 9, no. 1, p. 4, 2014.

[18] M. Zhang, T. Gao, J. Wang, J. Liao, Y. Qiu, Q. Yang, H. Xue, Z. Shi, Y. Zhao, Z. Xiong et al., "A hybrid fibers based wearable fabric piezoelectric nanogenerator for energy harvesting application," Nano Energy, vol. 13, pp. 298-305, 2015.

[19] C. Slouka, T. Kainz, E. Navickas, G. Walch, H. Hutter, K. Reichmann, and J. Fleig, "The effect of acceptor and donor doping on oxygen vacancy concentrations in lead zirconate titanate (pzt)," Materials, vol. 9, no. 11, p. 945,2016

[20] I. Johnston, D. McCluskey, C. Tan, and M. Tracey, "Mechanical characterization of bulk sylgard 184 for microfluidics and microengineering," Journal of Micromechanics and Microengineering, vol. 24, no. 3, p. 035017, 2014 
[21] Z. Wang, A. A. Volinsky, and N. D. Gallant, "Crosslinking effect on polydimethylsiloxane elastic modulus measured by custom-built compression instrument," Journal of Applied Polymer Science, vol. 131, no. 22, p. 41050, 2014

[22] J. Chun, N.-R. Kang, J.-Y. Kim, M.-S. Noh, C.-Y. Kang, D. Choi, S.-W. Kim, Z. L. Wang, and J. M. Baik, "Highly anisotropic power generation in piezoelectric hemispheres composed stretchable composite film for self-powered motion sensor," Nano Energy, vol. 11, pp. 1-10, 2015.

[23] X. Chen, "Fabrication and characterization of pzt nanofibers and their composites," Ph.D. dissertation, Stevens Institute of Technology, 2012.

[24] I. Babu and G. de With, "Highly flexible piezoelectric 0-3 pzt-pdms composites with high filler content," Composites Science and Technology, vol. 91, pp. 91-97, 2014.

[25] T. Yamada, T. Ueda, and T. Kitayama, "Piezoelectricity of a high-content lead zirconate titanate/polymer composite," Journal of Applied Physics, vol. 53, no. 6, pp. 4328-4332, 1982.

[26] A. Matei, V. Țucureanu, P. Vlăzan, I. Cernica, M. Popescu, and C. Romanitan, "Study of piezoelectric filler on the properties of pztpvdf composites," in AIP Conference Proceedings, vol. 1916, no. 1. AIP Publishing, 2017, p. 030006.

[27] C. Dias and D. Das-Gupta, "Poling behaviour of ceramic/polymer ferroelectric composites," Ferroelectrics, vol. 157, no. 1, pp. 405-410, 1994.

[28] D. Ponnamma, M. M. Chamakh, K. Deshmukh, M. B. Ahamed, A. Erturk, P. Sharma, and M. A.-A. Al-Maadeed, "Ceramic-based polymer nanocomposites as piezoelectric materials," in Smart Polymer Nanocomposites. Springer, Switzerland, 2017, pp. 77-93.

[29] H. Chan, Y. Chen, and C. Choy, "A poling study of pzt/p (vdf-trfe) copolymer 0-3 composites," Integrated Ferroelectrics, vol. 9, no. 1-3, pp. 207-214, 1995.

[30] A. Chiolerio, P. Rivolo, S. Porro, S. Stassi, S. Ricciardi, P. Mandracci, G. Canavese, K. Bejtka, and C. F. Pirri, "Inkjet-printed pedot: Pss electrodes on plasma-modified pdms nanocomposites: Quantifying plasma treatment hardness," Rsc Advances, vol. 4, no. 93, pp. 51477-51 485, 2014.

[31] C.-Y. Li and Y.-C. Liao, "Adhesive stretchable printed conductive thin film patterns on pdms surface with an atmospheric plasma treatment," ACS applied materials \& interfaces, vol. 8, no. 18, pp. 11 868-11 874, 2016.

[32] Y. Zhang, W. Zhu, C. K. Jeong, H. Sun, G. Yang, W. Chen, and Q. Wang, "A microcube-based hybrid piezocomposite as a flexible energy generator," RSC Advances, vol. 7, no. 52, pp. 32 502-32 507, 2017.

[33] H. Zhai, Y. Jiang, H. Li, P. Zhang, Y. He, D. Shi, X. Zhang, and J. Yang, "In situ polarization and dielectric property measurements of pb (zr0. 52ti0. 48) o3 ferroelectric nanocrystals," Heliyon, vol. 3, no. 6 , p. e00313, 2017.

[34] P. Sampathkumar, P. Gowdhaman, S. Sundaram, and V. Annamalai, "A review on pzt-polymer composites: dielectric and piezoelectric properties," Nano Vision, vol. 3, no. 3, pp. 223-230, 2013.

[35] S. Banerjee and K. Cook-Chennault, "An investigation into the influence of electrically conductive particle size on electromechanical coupling and effective dielectric strain coefficients in three phase composite piezoelectric polymers," Composites Part A: Applied Science and Manufacturing, vol. 43, no. 9, pp. 1612-1619, 2012.

[36] Y. Zhang, Z. Liu, F. Ding, and W. Zhang, "Effect of piezoelectric ceramic particles size gradation on piezoelectric properties of 0-3 cement-based piezoelectric composites," Smart Materials and Structures, vol. 27, no. 8, p. 085029, 2018.

[37] N. Ramdani, Polymer and Ceramic Composite Materials: Emergent Properties and Applications. CRC Press, 2019.

[38] S. K. Mahadeva, K. Walus, and B. Stoeber, "Piezoelectric paper for physical sensing applications," in 2015 28th IEEE International Conference on Micro Electro Mechanical Systems (MEMS). IEEE, 2015, pp. 861-864.

[39] C. Sakaki, B. L. Newalkar, S. Komarneni, and K. Uchino, "Grain size dependence of high power piezoelectric characteristics in nb doped lead zirconate titanate oxide ceramics," Japanese journal of applied physics, vol. 40, no. 12R, p. 6907, 2001.

[40] "Navy type pzt classifications," https://sensortechcanada.com/technicalnotes/articles/navy-type-pzt, accessed: 2019-12-13.

[41] T. Radhakrishnan, "Thermal degradation of poly (dimethylsilylene) and poly (tetramethyldisilylene-co-styrene)," Journal of applied polymer science, vol. 99, no. 5, pp. 2679-2686, 2006.

[42] H. J. Lim, M. K. Kim, H. Sohn, and C. Y. Park, "Impedance based damage detection under varying temperature and loading conditions," Ndt \& E International, vol. 44, no. 8, pp. 740-750, 2011.
[43] C. Fendzi, M. Rebillat, N. Mechbal, M. Guskov, and G. Coffignal, "A data-driven temperature compensation approach for structural health monitoring using lamb waves," Structural Health Monitoring, vol. 15, no. 5 , pp. 525-540, 2016.

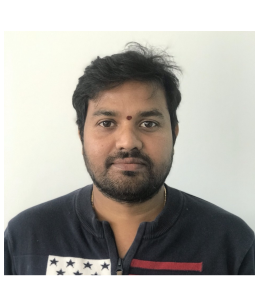

Kiran kumar Sappati received his B.Eng. from Andhra Univeristy, India, in 2006 and M.Tech from Indian Institute of Technology, Roorkee, India in 2008. He worked for Bharat heavy electricals limited, India from 2008-2017 in the field of controls and instrumentation. Mr. Sappati received spacial appreciations and rewards for his outstanding contribution in various projects. Currently he is pursuing his $\mathrm{Ph} . \mathrm{D}$. degree in electrical engineering from McGill University, Canada. His research interests are sensors and instrumentation, especially using piezoelectric materials. Currently, he is working on developing low cost flexible piezoelectric substrates for tailor made applications. Mr. Sappati is a recipient of MEDA award at McGill University.

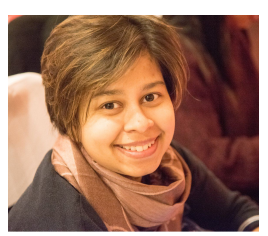

Sharmistha Bhadra received the B.Sc. degree in computer engineering from the University of New Brunswick, Fredericton, NB, Canada, and the M.Sc. and $\mathrm{Ph} . \mathrm{D}$. degrees in electrical engineering from the University of Manitoba, Winnipeg, MB, Canada. From 2015 to 2016 she was an NSERC postdoctoral fellow at the University of British Columbia, Vancouver, BC, Canada. She joined McGill University in 2016 and is currently an assistant professor. She has published numerous papers and holds 2 patents in sensor area. Her current research interests are in the area of printed and flexible hybrid electronics, microelectromechanical systems, and sensors and actuators. 